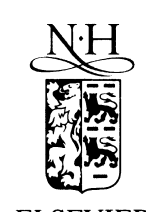

Nuclear Instruments and Methods in Physics Research B 135 (1998) 45-49

\title{
Contribution of nuclear scattering to the energy loss distribution of protons in carbon foils
}

\author{
Cristian D. Denton ${ }^{\mathrm{a}, *}$, Rafael Garcia-Molina ${ }^{\mathrm{b}}$, Isabel Abril ${ }^{\mathrm{a}}$, Néstor R. Arista ${ }^{\mathrm{c}}$ \\ a Departament de Física Aplicada, Universitat d'Alacant, Apartat 99, E-03080 Alacant, Spain \\ b Departamento de Física, Universidad de Murcia, Apartado 4021, E-30080 Murcia, Spain \\ ${ }^{\mathrm{c}}$ Instituto Balseiro, Centro Atómico Bariloche, RA-8400 Bariloche, Argentina
}

\begin{abstract}
We have developed a simulation code to study the energy loss distribution of energetic protons after traversing a thin amorphous carbon foil. The electronic interactions among the proton and the target electrons were taken into account using a realistic description of the solid, and the nuclear scattering events were considered by means of a Monte Carlo procedure. Our results compare fairly well with experimental energy loss distributions. (C) 1998 Elsevier Science B.V.
\end{abstract}

Keywords: Energy loss; Stopping power

\section{Introduction}

The interaction of energetic charged projectiles with matter goes back to the beginning of this century. Many references are available that describe the basic processes that take place in these interactions, from the classical Bohr's paper [1], to recent proceedings [2] or textbooks [3,4]. However there are still several aspects of the processes involved in these interactions that deserve further analysis.

When an energetic projectile moves through a solid it interacts with the target electrons (via collective and individual excitations) and also with the target nuclei (nuclear collisions). The former way of interaction takes place through inelastic processes and mainly changes the projectile kinetic energy, without modifying appreciably its direc-

\footnotetext{
*Corresponding author. E-mail: denton@ua.es.
}

tion; on the other hand, the series of elastic collisions with the atomic cores modify the projectile's velocity direction, and also changes slightly its energy. For projectile energies $E \geqslant 10$ $\mathrm{keV}$, the energy losses due to electronic excitations dominate over those due to elastic nuclear collisions, therefore the latter are usually not taken into account in the evaluation of energy loss at these energies.

Due to the stochastic nature of the interactions that take place at the atomic level, the slowing down of a proton beam produces a distribution of energy losses, which is mainly characterized by the mean energy loss per path length (the stopping power), and by the fluctuations around this mean value (the energy loss straggling). Although in the energy range of interest in this work both magnitudes are dominated by electronic interactions, the purpose of this paper is to study the effects 
of nuclear collisions on the energy loss distributions, in particular with respect to the following points: an expected increase in the energy loss, due both to an enlargement in the proton path length (due to collisional deflections), and to the additional energy losses in elastic processes. Moreover, the effect of a stopping power and energy loss straggling that change as the proton energy varies (through the slowing down process) is also incorporated in our calculations.

In this paper we use a combination of Molecular Dynamics and Monte Carlo code to analyze the effects of nuclear collisions on the proton energy loss distribution. The effect of a stopping power and straggling that change as the proton energy varies inside the solid is also incorporated in our calculations.

\section{Simulation method and energy loss calculations}

For a random medium, the electronic stopping force and the nuclear collision terms are in principle of stochastic nature. Then, for a given incident energy, the former fluctuates following a Gaussian distribution around a mean value. This mean value and the variance of this distribution are, respectively, the stopping power and the energy loss straggling, and both can be calculated from the dielectric theory of the stopping power $[5,6]$. The elastic collision deflections fluctuate around the forward direction.

Here we have taken into account both processes. The trajectories of the protons in an amorphous carbon target were simulated using a Molecular Dynamics code (based on the Verlet algorithm [7]) to follow the continuous slowing down due to electronic interactions; and the nuclear collisions were incorporated into this code via a Monte Carlo subroutine.

At each time-step the corresponding stopping forces were statistically generated from a Gaussian distribution, whose mean value and variance were calculated from the dielectric formalism $[5,6]$. The energy loss function of the amorphous carbon target, which lies on the grounds of the dielectric theory of stopping power, was determined according to the procedure described in Refs. [8-10].
The proton mean free path between nuclear collisions and the corresponding polar angle of deflection (with respect to the direction of motion of the proton) were calculated using the method described by Zajfman et al. [11]. The azimuthal angle was taken from a uniform distribution between 0 and $2 \pi$.

Using this method, the trajectory of each proton is followed since it enters the target until it leaves it. After repeating this procedure for 20000 histories we obtain the energy loss distribution of the proton beam after traversing different foil thicknesses, and from these distribution we derive the corresponding mean energy loss value for each thickness.

\section{Results and discussion}

We show in Fig. 1(a) and (b) the calculated distributions of energy losses, in cases where nuclear collisions were or were not taken into account; the incident proton energies are $E=10 \mathrm{keV}$ and $E=25 \mathrm{keV}$ and the thickness of amorphous carbon foil is $265 \AA$. The electronic energy loss straggling was included in both cases. As it may be observed, the net effect of the nuclear collisions is to increase the mean energy loss, but they do not affect sizably the width of the distributions. These two effects are easy to understand in terms of the increase in the effective path length of the protons (due to multiple deflections from the otherwise straight trajectories), and considering also the almost negligible energy loss in the elastic collisions. Then, it seems evident that the effects of nuclear collisions will be more notorious in the case of low energies and thick targets.

In Fig. 2(a) and (b) we observe the effect of including or not the fluctuations in the electronic energy loss experienced by a proton at each timestep. Results for two proton energies $(E=25$ and $100 \mathrm{keV})$ and several target thicknesses $(159,317$ and $529 \AA$ ) are depicted. Nuclear collisions, which were kept in both cases, produce a slight asymmetry of the distribution towards larger energy losses, but this is only appreciable when the electronic straggling is not included in the calculations. Again, it is clearly perceptible that the contribu- 

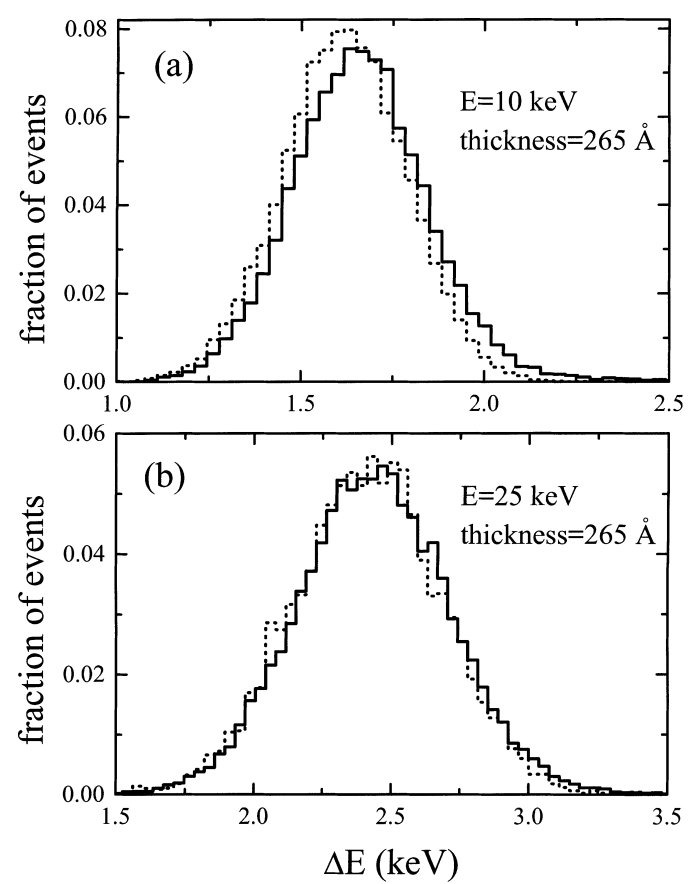

Fig. 1. Energy loss distribution calculated for a proton beam with energy: (a) $E=10 \mathrm{keV}$ and (b) $E=25 \mathrm{keV}$, after traversing a $265 \AA$-thick amorphous carbon foil. The solid and dashed lines correspond to calculations done taking or not into account, respectively, nuclear collisions in our simulations.

tion of the nuclear scattering to the energy loss distribution decreases for thinner targets and higher proton energies.

A comparison of our results with the experimental energy distribution of a $56.3 \mathrm{keV}$ proton beam transmitted through a $2.18 \mu \mathrm{g} / \mathrm{cm}^{2}$ amorphous carbon foil [12] is provided in Fig. 3. According to the technique used to prepare the target [12] we have considered that the density of this sample was $1.7 \mathrm{~g} / \mathrm{cm}^{3}$, which gives a foil thickness of $128 \AA$. The position and width of the peak predicted by our analysis agree quite well with the experimental results.

Fig. 4 shows the mean energy loss as a function of the foil thickness, for two proton energies $(E=10$ and $25 \mathrm{keV})$. Solid lines correspond to the case where nuclear collisions were taken into account in the simulations, whereas the opposite is represented by the dotted lines. Note that all the previous results were obtained allowing for the variation of the stopping power and straggling
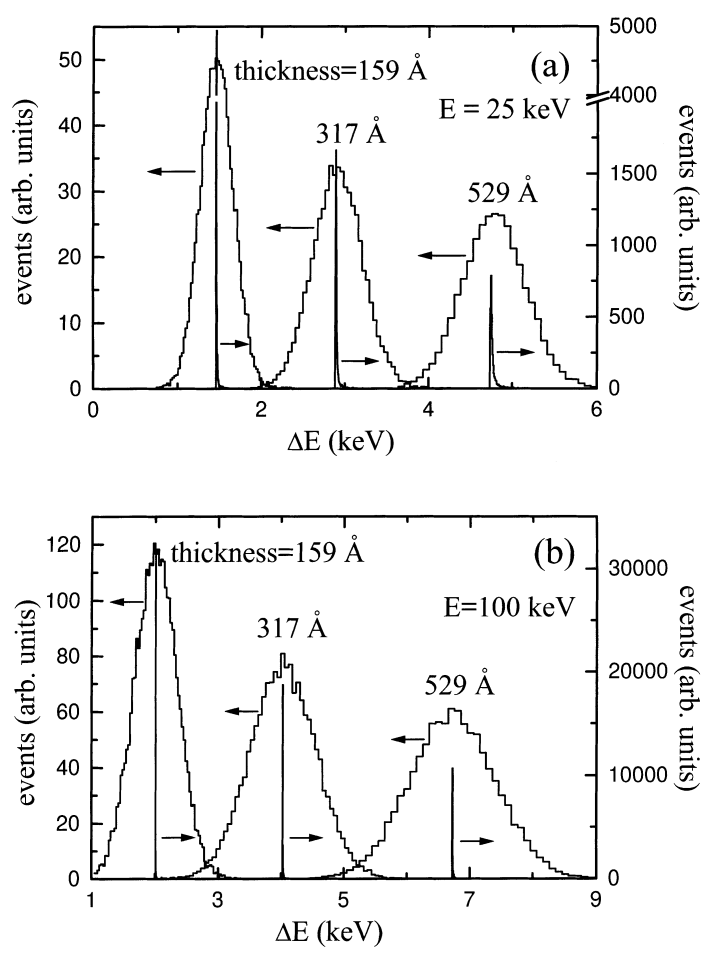

Fig. 2. Energy loss distributions calculated for two proton energies $(25$ and $100 \mathrm{keV})$, and for several amorphous carbon foil thicknesses (159, 317 and $529 \AA$ ). Broad distributions (left axis): with electronic energy loss straggling. Narrow distributions (right axis): without electronic energy loss straggling. Nuclear collisions are taken into account in both cases.

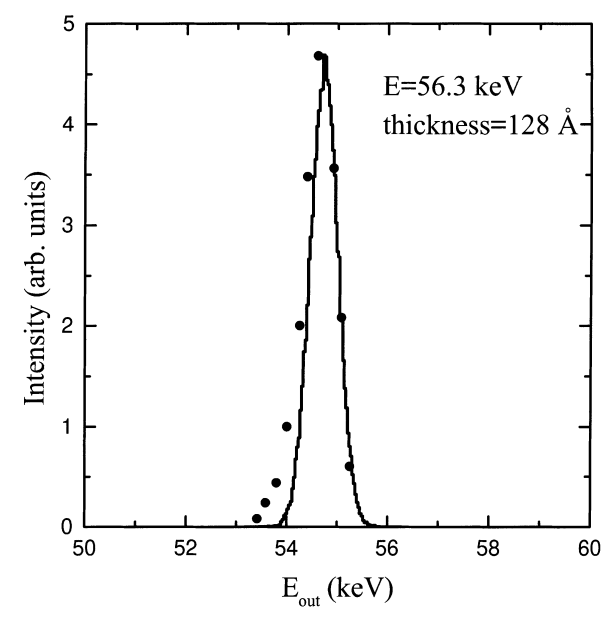

Fig. 3. Energy distribution resulting from the passage of a 56.3 keV-energy proton beam through a $128 \AA$ A-thick amorphous carbon foil. The solid line represents our calculations, and the dots are experimental data from [12]. 


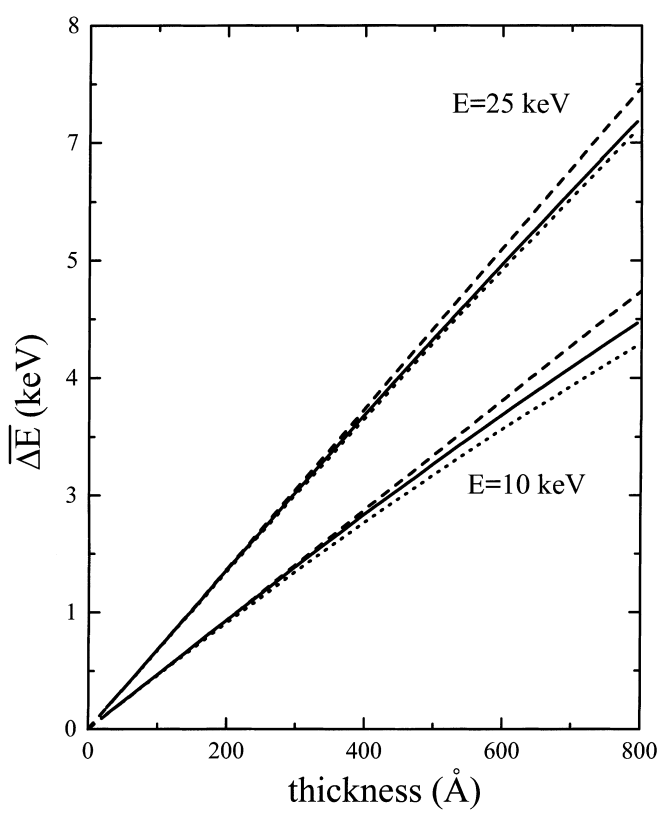

Fig. 4. Mean energy loss as a function of the target thickness, for two proton energies: (a) $E=10 \mathrm{keV}$ and (b) $E=25 \mathrm{keV}$. Solid lines: nuclear collisions were included in the calculations; dotted lines: nuclear collisions were not included in the calculations; dashed lines: nuclear collisions were not included in the calculations and a constant stopping power was used.

according to the instantaneous energy of the proton inside the foil. In order to check the convenience of the latter statement, we have also considered the simplest case of a constant stopping power (corresponding to the incident energy) without nuclear collisions, which is represented by the dashed lines. Here we can observe that the more complete calculations (solid lines) clearly differ from the simplest case (dashed line). The intermediate procedure, which considers the variation of the stopping power with energy, but does not include the nuclear collisions (dotted line), hardly differs from the complete calculations in the case of high energies, but it shows appreciable differences for lower energies and thicker targets.

\section{Conclusions}

We have developed a simulation code to calculate the energy loss distribution of proton beams interacting with solid targets. The effects of the electronic energy loss straggling, stopping power and straggling variation with the instantaneous proton velocity, and nuclear collisions, were taken into account. The first two effects, by following, in a continuous slowing down approximation, the motion of a proton through the solid, and the latter by using a scattering cross section to calculate the deflections of the protons from the initial straight trajectory.

The thickness dependence of the stopping power (calculated as the slope of the curve $\overline{\Delta E}$ vs. thickness) is worth noting. The usual procedure to obtain experimentally the stopping power, by measuring the energy losses for different foil thicknesses should be handled carefully, at least in the range of energies and thicknesses discussed in this paper. These differences are larger when the thicker is the target, and the lower the incident proton energy.

\section{Acknowledgements}

We acknowledge the Spanish Dirección General de Investigación Científica y Técnica (projects PB95-0689 and PB96-1118) for financial support, the Instituto de Cooperación Iberoamericana for a grant to CDD, and the Conselleria d'Educació i Ciència de la Generalitat Valenciana for a visiting professor fellowship to NRA under the program PROPIO.

\section{References}

[1] N. Bohr, K. Dans. Vidensk. Selsk. Mat. Fys. Medd. 18 (8) (1948).

[2] A. Gras-Martí, H.M. Urbassek, N.R. Arista, F. Flores (Eds.), Interaction of Charged Particles with Solids and Surfaces, NATO ASI Series, vol. B 271, Plenum, New York, 1990.

[3] M.A. Kumakhov, F.F. Komarov, Energy Loss and Ion Ranges in Solids, Gordon and Breach, New York, 1981.

[4] V.V. Balashov, Interaction of Particles and Radiation with Matter, Springer, Berlin, 1997.

[5] N.R. Arista, Phys. Rev. B 18 (1978) 1.

[6] P.M. Echenique, R.H. Ritchie, W. Brandt, Phys. Rev. B 20 (1979) 2567.

[7] M.P. Allen, D.J. Tildesley, Computer Simulations of Liquids, Oxford Science Publications, Oxford, 1987. 
[8] I. Abril, R. Garcia-Molina, N.R. Arista, Nucl. Instr. and Meth. B 90 (1994) 72.

[9] D.J. Planes, R. Garcia-Molina, I. Abril, N.R. Arista, J. Electr. Spectrosc. Relat. Phenom. 82 (1996) 23.

[10] I. Abril, R. Garcia-Molina, C.D. Denton, F.J. PérezPérez, N.R. Arista, Phys. Rev. A (in press).
[11] D. Zajfman, G. Both, E.P. Kanter, Z. Vager, Phys. Rev. A 41 (1990) 2482.

[12] J.H. Ormrod, H.E. Duckworth, Can. J. Phys. 41 (1963) 1424. 